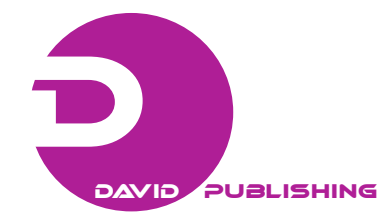

\title{
Elastic-Plastic Transition under Uniaxial Stress BCC
}

\section{Tantalum}

\author{
Oscar Guerrero and Marcelo Marucho \\ Department of Physics and Astronomy, University of Texas at San Antonio, San Antonio 78249-0697, Texas
}

Received: October 02, 2012 / Accepted: November 08, 2012 / Published: March 25, 2013.

\begin{abstract}
Classical molecular dynamics and lattice dynamics were utilized to study the elastic-plastic transition underuniaxial stress in defect free body center cubic (BCC) Tantalum crystals. We demonstrate that the nucleation of defects at the time scales of molecular dynamics in tantalum is due to dynamical instabilities (soft-phonons). Uniaxial compressions test were simulated along the crystallographic directions (100), (110) and (111). The results show that the nucleation of defeccts in the direction (110) is due to crystal twinning.
\end{abstract}

Key words: BCC crystals, crystal defects, tantalum crystals, soft-phonons, molecular dynamics simulations, elasticplastic crystal transitions.

\section{Nomenclature}

$\begin{array}{ll}\text { CRSS: } & \text { Critical resolved shear stress } \\ \text { FCC: } & \text { Face-centered cubic } \\ \text { BCC: } & \text { Body-centered cubic } \\ \text { EAM: } & \text { Embedded atom method } \\ \text { MD: } & \text { Molecular Dynamics } \\ \text { BZ: } & \text { Brillouin Zone }\end{array}$

\section{Greek letters}

$t: \quad$ Shear stress deformation

\section{Introduction}

Theoretical and experimental determination of the mechanical properties of metallic materials under high pressures takes place in important research fields including material science, solid-state physics, geophysics, and planetary physics. It is well known that yield strength and ductility, as well as the elasticity of metals are strongly affected by even moderate pressures. When a perfect single crystal is deformed beyond its elastic limit, defects are generated which allows plastic flow. This can be seen as the ideal

Corresponding author: Marcelo Marucho, Ph.D., assistant professor, research field: soft and condensed matter. E-mail: marcelo.marucho@utsa.edu. strength of a material which is an upper-bound of what can be obtained practically. Despite the considerable volume of interest that this problem has generated, as yet there does not seem to be a common view as to the underlying mechanism behind the defect generation. Several models around the year 1930 were proposed in perfect crystals to estimate the maximum stress before inducing plasticity $[1,2]$. At the atomic level, the plasticity is described by the rearrangement of atoms from its current configuration. The plastic anisotropy is caused by preferred planes where dislocations move (slip planes) [3]. In 1926, Frenkel [4] stated that a critical value of shear is necessary to move the atoms in a layer over another. In 1934, Taylor [5] found out that a critical resolved shear stress (CRSS) is the same for all the slip systems in a given crystal. In 1951, Bishop and Hill [6] explained that the amount of plastic work per unit volume is a maximum. The above mentioned models, and in general all the structural models in civil engineering and materials science, use the stress (force/area) as the primary measure of plastic deformation and mechanical strength of a material, i.e., the defect nucleation occurs when the resolved shear stress reaches a critical value. 
However, the results obtained from simulations of shock compression molecular dynamics in FCC Copper (100) are not in agreement with the aforementioned description [7, 8]. These simulations show that the yield strength occurs at a shear stress below the maximum of the specified direction and at a strain higher than those produced by the critical stress value. Fig. 1 shows the theoretical curve of the shear $\tau$ $=0.5\left(\sigma_{z z}-0.5\left(\sigma_{x x}+\sigma_{y y}\right)\right)$ vs uniaxial deformation along the crystallographic direction (110) for Tantalum [9]. Indeed, the elastic limit occurs at a strain higher than those produced by the critical shear.

This analysis gives the hint that these models related to the calculation of the critical yield stress at the time scale of molecular dynamics might be inappropriate and other mechanisms would be responsible for the nucleation of defects in BCC perfect metallic crystals. An alternative description of elastic-plastic transitions is given by the soft-mode theory of phase transitions in terms of an anomalous behavior of the elastic modulus under compression above Mbar pressure [10-14]. This anomaly originates from a particular combination of stresses and strains around a defect that generate a lattice vibrational mode to soften significantly in the region near the defect and serves as a center for nucleation [15]. This phenomenon has been observed in Martensitic transformations in which diffusionless (meaning no atom by-atom rearrangement), displacive transitions (meaning small cooperative motions of atoms) alter the symmetry but without changing the composition or order [16, 17].

Therefore, a detailed understanding of the anomalous behavior of the elastic modulus is essential to elucidate the mechanisms governing the defect generation in BCC perfect metallic crystals. A crucial step in arriving at this understanding is to establish the fundamental relationship between the phonon dispersion and the yield strain. In pursuit of this challenge, we studied in this article the elastic-plastic transition in perfect BCC crystal tantalum at low temperature under uniaxial stress load and extreme

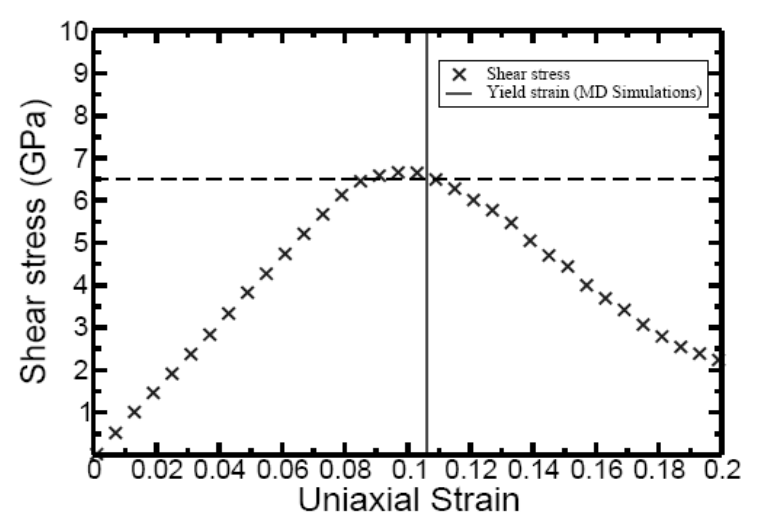

Fig. 1 EAM theoretical curve of shear stress ( $t$ ) vs. strain along (110) at $0 \mathrm{~K}$. The line indicates where the elastic-plastic transition occurs using molecular dynamics simulations. The black dashed line indicates the maximum shear.

conditions: Megabar pressures and high strain rates (> $\left.108 \mathrm{~s}^{-1}\right)$ [18].

\section{Model}

We chose tantalum because it is one of the most widely used benchmarks for the study of yield strength and for testing theoretical predictions of elastic and plastic behavior under extreme conditions [19-22]. It is BCC at room pressure and temperature and it is predicted to retain the BCC structure over a very large pressures and temperature range [23]. Using this model we performed molecular dynamics (MD) simulations based on embedded atom method (EAM) [24] to study the mechanical stability over a large temperature range and along different directions of uniaxial compression (Fig. 2). Interestingly, the results presented in this article are not potential-dependent. We obtained a nucleation defect generation along the (110) direction characterized by non temperature-dependent critical strains. At temperature of $300 \mathrm{~K}$, the instant of onset of nucleation was obtained by deforming the crystal using a compression test (Fig. 3). Once the elastic plastic transition was identified, the defects began to grow until they filled the computational box completely (Fig. 4). The morphology of the defects in the direction (110) was identified as crystal twinning [25]. Finally, the phonon-dispersion curve as a function of 


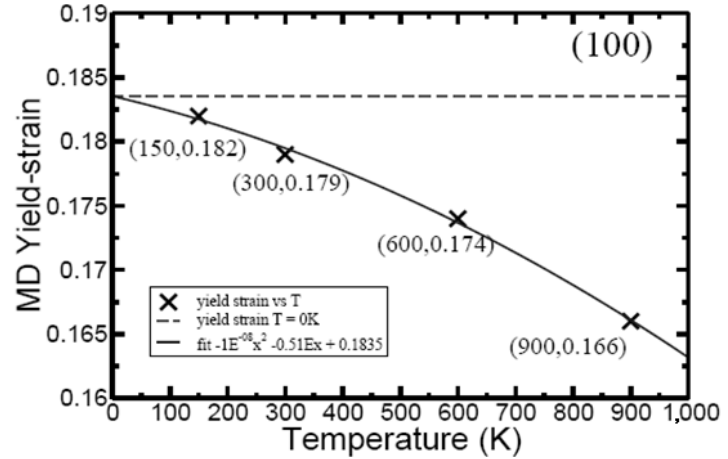

(a)

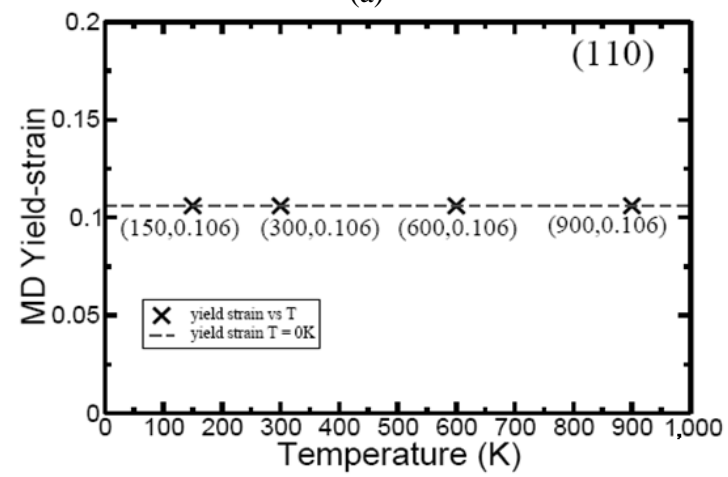

(b)

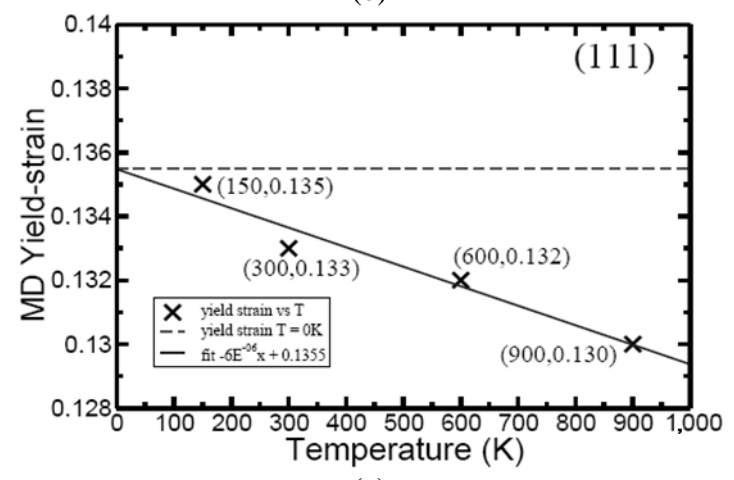

(c)

Fig. 2 Yield strain as function temperature along the different directions of uniaxial compression. (a) (100), (b) (110), (c) (111). the yield strains was calculated. In doing so, the speed of sound of an uniaxial strained lattice is obtained by means of the numerical evaluation of the dynamical matrix and subsequently correlated along different directions with the elastic limit threshold obtained from MD simulations (Figs. 5 and 6). We found out that these dynamical instabilities (soft-phonons) play an important role in the elastic-plastic transition in BCC tantalum crystals. We demonstrate that the elastic-plastic transition in BCC tantalum crystals at low temperature and uniaxial compression can be described in terms of soft (unstable) phonon modes, which produce defect nucleation growing on nanosecond timescales. The characteristics of these soft modes, in terms of their complex frequencies and distribution within the brillouin zone (BZ) as a function of compression, explains several key aspects of elastic-plastic transition in BCC crystals that have been a matter of debate for some years. Conceptually, similar phenomena have been invoked to explain the observed rhombohedral distortion that occurs in vanadium [26] and the temperature dependence of the elastic moduli of niobium [27]. However, while theory elements [28-30], no experiments have indicated the presence of an anomalous softening of the transverse-acoustic phonons in Ta. We show why defect generation occurs not, as might naivel bethought, simply above a critical shear stress, but at acertain stress and strain (Fig. 2). Importantly, the fact that the unstable regions are not at high symmetry positions of

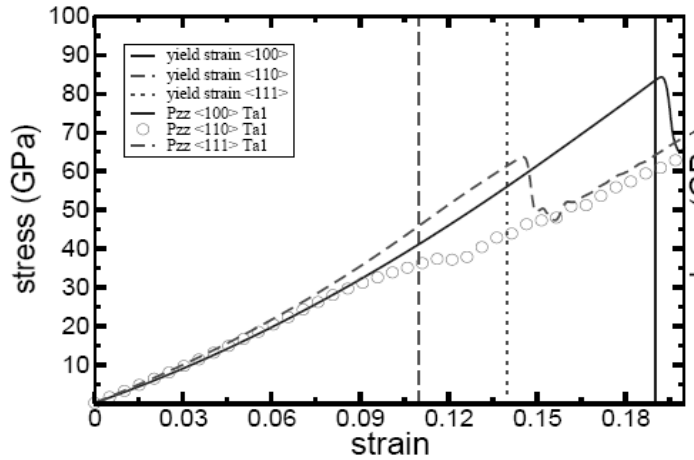

(a)

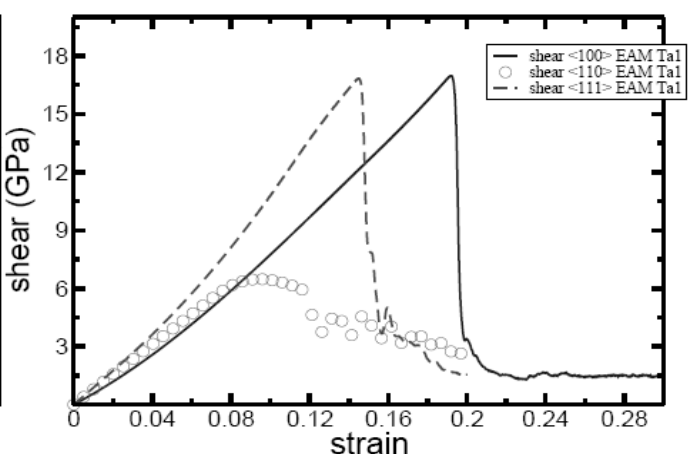

(b)

Fig. 3 (a)Stress vs strain curve for uniaxial compression and (b) curve of shear (t) vs. uniaxial compression at $300 \mathrm{~K}$, that was obtained using molecular dynamics along the directions (100), (110) and (111). 


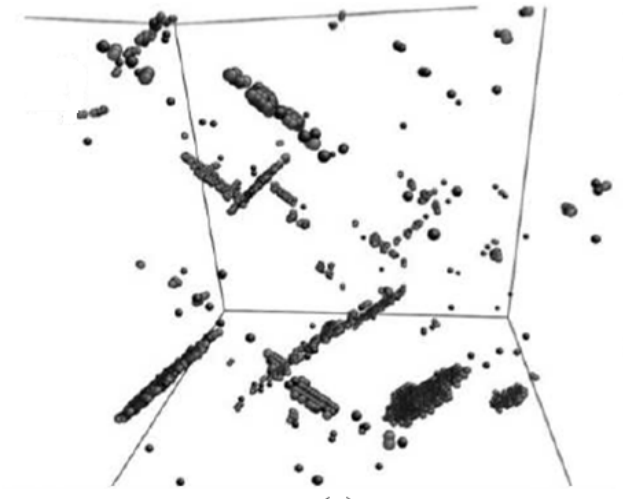

(a)

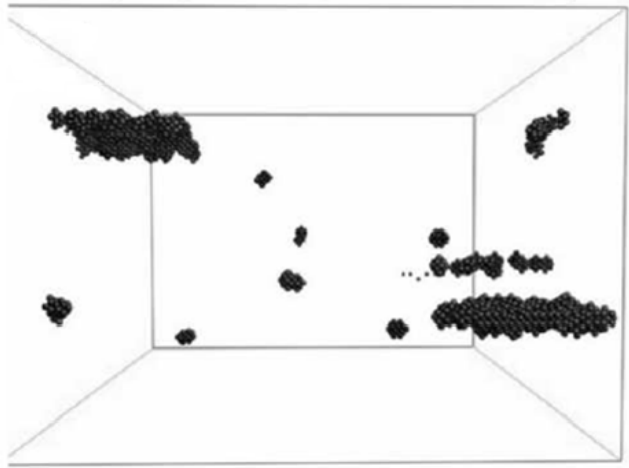

(c)

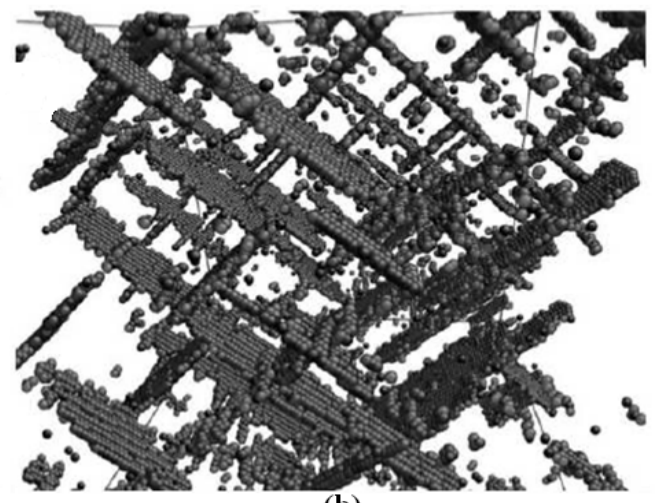

(b)

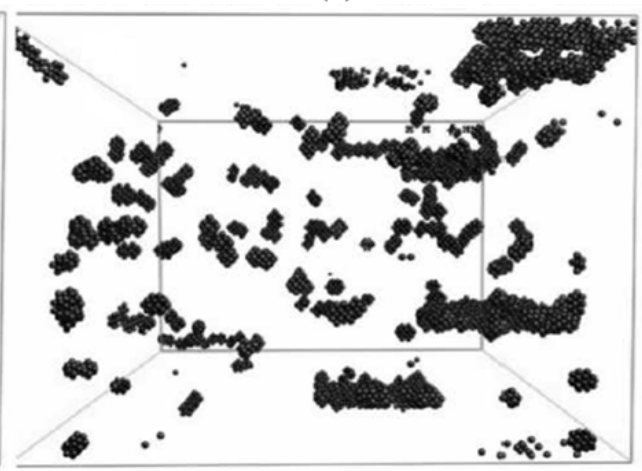

(d)

Fig. 4 (a) and (b) Simulation of 18 million atoms along (110) at $300 \mathrm{~K}$ and at a strain of $11 \%$. (c) and (d) Simulation of 16 million atoms along (100) at $300 \mathrm{~K}$ and at a strain of $19 \%$. These images show the start and final stage of the defect nucleation.

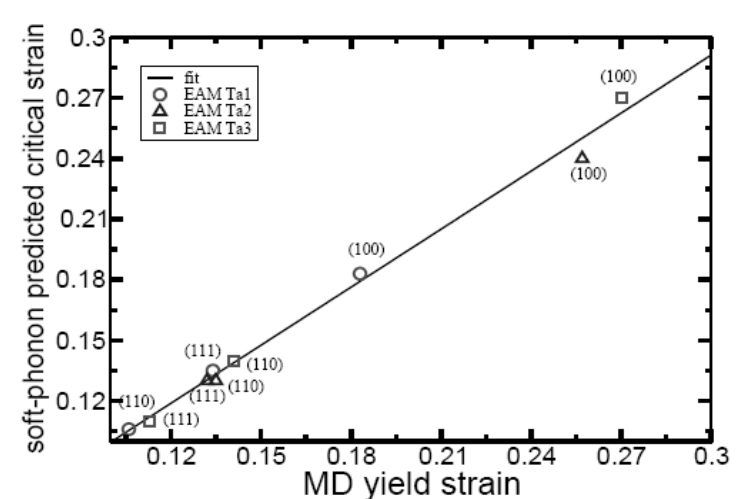

Fig. 5 A correlation between critical strain determined by the dynamic instability $|v s|=0$ and the yield determined from molecular dynamics simulations using different EAM models along the different directions of uniaxial compression.

the BZ further explains why certain simulation box sizes are required to observe plasticity at certain values of compression, and how the box size determines the time scale for defects to be generated. Furthermore, the wavelength of the instabilities determines the periodicity of the defects generated and hence influences the defect density and strength of the material.

In the next sections, we describe the numerical calculations and we analyze the results. The last section is left for the conclusions.

\section{Molecular Dynamics Simulations of the Elastic-Plastic Transition in Tantalum}

The molecular dynamics simulations were performed using the LAMMPS program [31]. We used three new developed EAM potential interactions named Ta1, Ta2 and Ta3 [9, 10] these EAM interatomic potentials of Tantalum were created with the goal of extending the range of transferability of the model with no solid-solid phase transitions within 0-200 GPa $(T=0)$. The fitting database included both experimental and density functional theory (DFT) data: lattice constant, cohesive energy, unrelaxed vacancy formation energy, elastic constants, equation of state (EOS) of various phases: BCC, FCC, HCP and A15. 
Details on the functional forms and quantitative comparisons with experimental and DFT data will be provided in a forthcoming publication. All the EAM models (Ta1, Ta2, Ta3) reproduced the equation of state (EOS), bulk modulus and approximately same elastic constant as the experimental data for Tantalum [32]. However, the energy-displacement surfaces along preferred low index directions (Gamma-Surface) were different. The figures in this paper correspond to the Ta1 potential. The use of Ta2 and Ta3 potentials leaded to the same nucleation mechanism, however, the yield strain occurred in other percentage due to the change in the energy-displacement surfaces [34, 35]. The simulations were performed as follows. First a BCC crystal with a lattice parameter of 3.304 Angstroms was created, the boundary conditions of the computational box being periodic. Next, an equilibration step was performed by using the isothermal-isobaric (NPT) [36] ensemble that allows

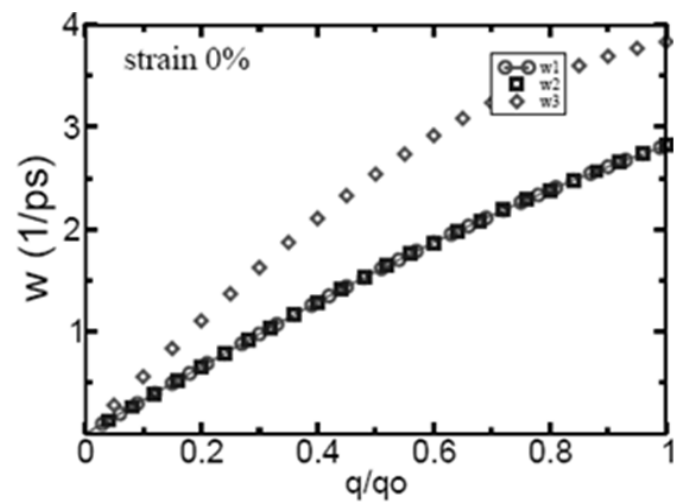

(a)

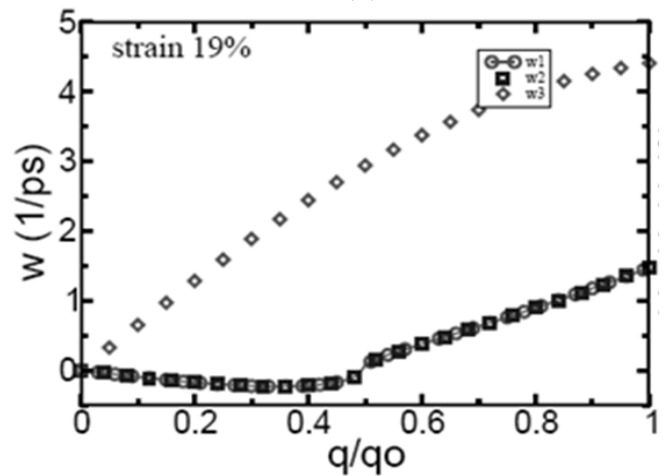

(c) the lattice to expand to a temperature of $300 \mathrm{~K}$ with a pressure of $0 \mathrm{kbar}$ at each simulation cell boundary. Subsequently, the simulation cell is deformed isothermally in the $\mathrm{x}$ direction at a strain rate of 0.005 $1 / \mathrm{ps}$, while the lateral boundaries ( $\mathrm{y}$ and $\mathrm{z}$ ) are controlled by using the NVT equations of motion to maintain the length of the computational box constant [36]. The stress and strain values are output to a separate file, which can be imported to a graphing application for plotting. CFG files [37] were dumped, which includes the $\mathrm{x}, \mathrm{y}$, and $\mathrm{z}$ coordinates, the centrosymmetric values, the potential energies, and forces for each atom. This can be visualized using Atom Eye [37]. In these simulations, we used a time-step of 0.005 picoseconds.

The elastic-plastic transition was determined by a decrease in pressure (Fig. 3), a sudden increase in temperature and a drastic decline of the shear stress $\tau$. The same procedure was repeated for different value of the temperatures in the equilibration step, and

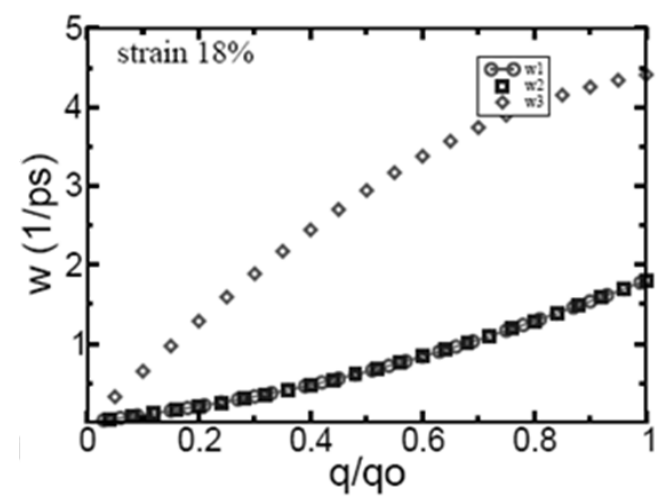

(b)

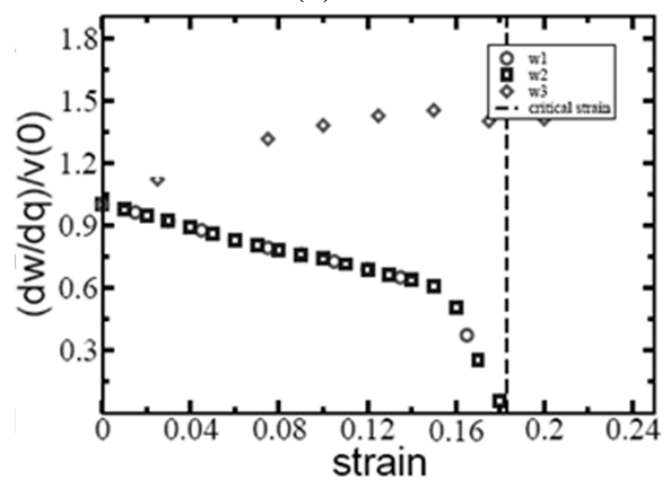

(d)

Fig. 6 The phonons dispersions for (a) $0 \%$; (b)18\% and (c) 19\% of compression, respectively. As the strain increases the Transverse phonon softening is characteristic of the instability, i.e., w2 = w3 = 0; (d) Numerical evaluation of Sound velocity [dw/dq/velocity(zero strain)] by means of the phonon dispersions for an uniaxial strained Ta crystal along (100) andwave propagation direction $<001>$. 
reorientation of the $\mathrm{x}$ axis of the computational cell along the crystallographic directions (110) and (111), respectively. Fig. 2 shows the elastic threshold obtained from MD simulations as function of temperature, and the extrapolated value up to zero Kelvin.

\subsection{Plasticity in Tantalum (110) and (100)}

The Nucleation of defects was visualized using the Centro symmetry parameter and the atomic local strain tensor coloring [37] for uniaxial deformation in the directions (110) and (100) respectively. For the direction (100) the separation of the atoms was compared with the initial configuration of a BCC crystal. It was of utmost importance to identify the exact moment where the nucleation of defect occurs. The instant of onset of nucleation was obtained by deforming the crystal using a compression test (Fig. 3). In doing so, first we identified elastic plastic transition and the corresponding critical yield strain. Next, we compressed the computational box up to the obtained critical yield strain value. Finally, we ran the simulations in the canonical ensemble (NVT) [36]. After a certain number of time steps, the nucleation of the defects was observed. By continuing the observation, the defects began to grow until they filled the computational box completely. Fig.4 shows the growth of defects in the direction (110) and (100). The morphology of the defects in the direction (110) was identified as crystal twinning [25].

\section{Evaluation of Dynamical Instabilities in Perfect Crystals}

The propagation of sound in a crystal is usually analyzed by finding the acoustic phonon dispersion curves. In doing so, the eigenvalues of the dynamical matrix $w(q)$ must be calculated [38-41]. For a given wave vector number $\hat{q}$, three acoustic waves with mutually perpendicular polarizations can propagate. Waves with polarization vectors parallel or perpendicular to the direction of propagation are called longitudinal or transverse, respectively. At different low-index directions of the Brillouin zone and at temperature of $0 \mathrm{~K}$, we calculated the phonon spectra for an uniaxial strained BCC Tantalum lattice along (100), (110) and (111), respectively. Among all the possible directions of propagation $\hat{q}$ that an elastic wave can travel, we choose the one in which the condition $|v s|=0$ is found at the lowest strain. The same method was implemented for the three EAM potentials. The condition of dynamical instability was correlated with the elastic plastic threshold obtained from molecular dynamics simulations along the different directions of compression (Fig. 2). The correlation demonstrates that the elastic limit occurs when the condition of dynamic instability is satisfied regardless of the orientation and potential interaction (Fig. 5). The evaluation of the sound velocity by means of the phonon dispersions for an uniaxial strained tantalum crystal along (100) and wave propagation direction $\hat{q}=<001>$ is shown in Fig. 6. As the strain increases, the slope of the transverse modes of vibration (acoustic phonon unstable branches) $(\mathrm{dw} / \mathrm{dq}) \mid \mathrm{q}=0$ tends to zero. As a result, the transverse speed vs of sound along certain crystallographic directions in the crystal lattice vanishes i.e., $|v s|=0$. For all the EAM tantalum models, the nucleation of defects was never observed at strains below the soft-mode dynamical instability critical strain.

\section{Conclusions}

Classical molecular dynamics and lattice dynamics were utilized to study the elastic-plastic transition under uniaxial stress in defect free BCC Tantalum crystals. The large anisotropy in the critical stress in the observed defect formation reveals that the resolved stress (CRSS) and the formulation of Taylor does not apply. This is not surprising given the well known violation of Schmidt law in these materials. The uniaxial compressions test simulated along the crystallographic directions (100), (110) and (111) show that the nucleation of defects in the direction (110) is due to crystal twinning. The behavior of the phonon 
dispersion as a function of strain demonstrates that the nucleation of defects at the time scales of molecular dynamics in Tantalum is due to dynamical instabilities (soft-phonons). A knowledge of the time-scale and means by which defects nucleate, and how this varies with temperature, could be of importance in the simulation of other crystal structures such as twinned FCC metals which show a strong surface effect for defect nucleation [42, 43].

\section{Acknowledgments}

The work presented in the article was part of Oscar Guerrero's thesis under the supervision of Dr. Ramon Ravelo who provided the guidance and direction for the research.

\section{References}

[1] S.D. Shafrai, T.N. Hatsevich, Thermodynamic criterion for predicting the brittleness and plasticity of metals, Journal of Applied Mechanics and Technical Physics 40 (1999) 1148.

[2] J.A. Zimmerman, H. Gao, F.F. Abraham, Generalized stacking fault energies for embedded atom FCC metals, Modell. Simul. Mater. Sci. Eng. 8 (2000) 103.

[3] T. Yalcinkaya, W. Brekelmans, M. Geers, BCC single crystal plasticity modeling and its experimental identification, Modelling Simul. Mater. Sci. Eng. 16 (2008) 085007.

[4] J. Frenkel, On the theory of elasticity and yield stress in crystalline bodies, Z. Phys 37 (1926) 572. (German)

[5] G.I. Taylor, The mechanism of plastic deformation of crystals: Part 1. Theoretical, Theoretical. in: Proc. R. Soc., London, A145, 1934, P. 362.

[6] J.W.F. Bishop, R. Hill, A theory of the plastic distortion of a polycrystalline aggregate undercombined stresses, Phil. Mag. 42 (1951) 414.

[7] B.L. Holian, P.S. Lomdahl, Plasticity induced by shock waves in no equilibrium molecular-dynamics simulations, Nature 80 (1988) 2085.

[8] E.M. Bringa, J.U. Cazamias, P. Erhart, J. Stölken, N. Tanushev, B.D. Wirth, et al., Atomistic shock Hugoniot simulation of single-crystal copper, J. Appl. Phys. 96 (2004) 3793.

[9] O. Guerrero, Pressure induced dynamical instabilities in body center cubic crystals, Thesis, M.S., University of Texas at El Paso (UTEP), 2010, p. 35.
[10] R. Ravelo, A. Qi, C.T. Germann, B. Holian, Large-scale molecular dynamics simulations of shock induced plasticity in tantalum single crystals, AIP Conf. Proc. 1426 (2011) 1263.

[11] D. Antonangeli, D.L. Farber, A.H. Said, L. R. Benedetti, C.M. Aracne, D. Antonangeli, et al., Shear softening in tantalum at Mbar pressure, Phys. Rev. B 82 (2010) 132101.

[12] D. Clatterbuck, C. Krenn, M. Cohen, J. Morris, Phonon instabilities and the ideal strength of aluminum, Phys. Rev. Lett. 91 (2003) 135501.

[13] J. Li, T. Zhu, S. Yip, K.J. Van Vliet, S. Suresh, Elastic criterion for dislocation nucleation, Mater. Sci. Eng A 365 (2004) 25.

[14] S.D. Pavlov, Phonon spectrum in strained alkali metal lattices, Soviet Physics Journal 11 (1968) 99-99.

[15] P.C. Clapp, Pseudopotential Band Structure of ZnO, Phys. Status Solidi B 57 (1973) 561; Localized soft modes and ultrasonic effects in first order displacive transformations, Mater. Sci. Eng. 38 (1979) 193.

[16] S.M. Shapiro, E.C. Svensson, C. Vettier, B. Hennion, Uniaxial-stress dependence of the phonon behavior in the premartensitic phase of $\mathrm{Ni}_{62.5} \mathrm{Al}_{37.5}$, Phys. Rev. B 48 (1993) 13223.

[17] S.M. Shapiro, Neutron scattering studies of pretransitional phenomena in structural phase transformations, Metall.Trans. 12A(1990567), Mater. Sci. Forum 33 (1981) 56.

[18] R. Ravelo, B.L. Holian, T.C. Germann, High strain rates effects in quasi-isentropic compression of solids, in: AIP Conf. Proc., 2009, p. 1195.

[19] G. Wang, A. Strachan, T. Cagin, W.A. Goddard III, The role of core polarization curvature of screw dislocations in determining the Peierls stress in bcc Ta, a new criterion for designing high performance materials, Phys. Rev. B 67 (R) (2003) 140101.

[20] A. Dewaele, P. Loubeyre, Mechanical properties of tantalum under high pressure, Phys. Rev. B72 (2005) 134106.

[21] Z.L. Liu, L.C. Cai, X.R. Chen, Q. Wu, F.Q. Jing, Ab initio refinement of the thermal equation of state for bcc tantalum: the effect of bonding on anharmonicity, J. Phys.: Condens. Matter 21 (2009) 095408.

[22] T.J. Vogler, On measuring the strength of metals at ultrahigh strain rates, J. Appl. Phys. 106 (2009) 053530.

[23] D. Orlikowski, P. Söderlind, J.A. Moriarty, First-principles thermoelasticity of transition metals at high pressure: Tantalum prototype in the quasiharmonic limit, Phys. Rev. B 74 (2006) 054109.

[24] M.S. Daw, M.I. Baskes, Embedded-atom method: Derivation and application to impurities, surfaces, and other defects in metals, Phys. Rev. B 29 (1984) 6443. 
[25] M.A. Meyers, L.E. Murr, Shock waves and high-strain-rate phenomena in metals, Plenum Press, New York, 1981, pp. 675-702.

[26] Y. Ding, R. Ahuja, J. Shu, P. Chow, W. Luo, H.K. Mao, Structural phase transition of vanadium at $69 \mathrm{GPa}$, Phys. Rev. Lett. 98 (2007) 085502.

[27] Y. Talmor, E. Walker, S. Steinemann, Elastic constants of nionium up to the melting point, Solid State Commun. 23 (1977) 649.

[28] L. Ko־ci, Y. Ma, A.R. Oganov, P. Souvatzis, R. Ahuja, Elasticity of the superconducting metals $\mathrm{V}, \mathrm{Nb}, \mathrm{Ta}, \mathrm{Mo}$, and W at high pressure, Phys. Rev. B 77 (2008) 214101.

[29] A. Landa, J. Klepeis, P. Söderlind, I. Naumov, O. Velikokhatnyi, L. Vitos, et al., Ab initio calculations of elastic constants of the bcc V-Nb system at high pressures, J. Phys. Chem. Solids 67 (2006) 2056.

[30] A. Landa, P. Söderlind, A.V. Ruban, O.E. Peil, L. Vitos, Stability in BCC transition metals: Madelung and band-energy effects due to alloying, Phys. Rev. Lett. 103 (2009) 235501.

[31] S.J. Plimpton, Fast parallel algorithms for short-range molecular dynamics, J Comp Phys 117 (1995) 1.

[32] J.M. Zhang, Y.N. Wen, K.W. Xu, Atomic simulation of the vacancies in BCC metals with MAEAM, Cent. Eur. J. Phys. 4 (2006) 481.

[33] L.J. Walpole, The elastic shear moduli of a cubic crystal, J. Phys. D: Appl. Phys. 19 (1986) 457.
[34] V.V. Bulatov, W. Cai, R. Baran, K. Kang, Geometric aspects of the ideal shear resistance in simple crystal lattices, Philosophical Magazine 86 (2006) 3847.

[35] W. Shinoda, M. Shiga, M. Mikami, Rapid estimation of elastic constants by molecular dynamics simulation under constant stress, Phys Rev B 69 (2004) 134103.

[36] S. Nose, A unified formulation of the constant temperature molecular dynamics methods, Chem. Phys. 81 (1984) 511.

[37] J. Li, Atom Eye: an efficient atomistic configuration viewer, Modelling Simul. Mater. Sci. Eng. 11 (2003) 173.

[38] D. Wallace, Thermodynamics of Crystals, Dover, New York, 1972, pp. 1-484.

[39] E.R. Cowley, Lattice dynamics of silicon with empirical many-body potentials, Phys. Rev. Lett. 60 (1988) 2379.

[40] G. Simonelli, R. Pasianot, E.J. Savino, Phonon-dispersion curves for transition metals within the embedded-atom and embedded-defect methods, Phys Rev B 55 (1997) 5570.

[41] J.R. Ray, Effective elastic constants of solids under stress: Theory and calculations for helium from 11.0 to $23.6 \mathrm{GPa}$, Phys. Rev. B 40 (1989) 423.

[42] Y. Wei, Anisotropic size effect in strength in coherent nanowires with tilted twins, Phys. Rev. B 84 (2011) 014107.

[43] X. Li, Y. Wei, L. Lu, K. Lu, H. Gao, Dislocation nucleation governed softening and maximum strength in nano-twinned metals, Nature 464 (2010) 877. 